F Faculty

A Aid to

A CPD Self-Assessment Test

C CPD

T Topics

ANSWER SHEET

Review No. 2001/01

To be reviewed not later than 31 October 2005

\title{
Hormonal contraception and migraine
}

1. The risk of ischaemic stroke is reduced with low dose COCs $(<350 \mu \mathrm{g}$ ethinyloestradiol) compared to high dose COCs (> $350 \mu \mathrm{g}$ ethinyloestradiol).

Answer

2. Low-dose COCs may be prescribed safely to smokers over the age of 35 .

True

3. The expected incidence of ischaemic stroke in women with migraine with aura taking a low dose COC is more than 10 times higher than the expected incidence of ischaemic stroke in women of the same age without migraine and not using COCs.

False

True

4. Smoking is not a significant risk factor for ischaemic stroke in women with migraine.

False

5. All young women with migraine are at high risk of ischaemic stroke.

False

6. COCs may be used with caution in a woman with migraine with aura if she has no more than one additional risk factor for stroke.

False

7. Depo-Provera should not be used by a woman who suffers from migraine with aura.

False

8. COCs containing third generation progestogens are associated with a lower risk of ischaemic stroke compared to COCs containing second generation progestogens.

False

9. Depression and food cravings are typical symptoms of migraine aura.

False

10. The evolution of migraine aura is slow, taking several minutes to spread to maximum distribution.

True 